\section{CAS Diakonie-Entwicklung - ein Weiterbildungsangebot zur Stärkung einer diakonischen Kirche}

Gregor Scherzinger

\section{Die Nähe zu den Menschen - eine Sorge der Kirchen vor der Zukunft}

Nicht umsonst überschneiden sich die Zukunftspapiere der katholischen und evangelisch-reformierten Kirche in der Region St. Gallen genau in der Frage, wie sich die Institution die Nähe zu den Menschen bewahren kann. Die evangelisch-reformierte Kirche im Kanton St. Gallen betitelt ihre Vision 2025 mit «nahe bei Gott - nahe bei den Menschen». Das Bistum St. Gallen hat seinen pastoralen Perspektiven und Grundhaltungen für die Zukunft drei Optionen zum Thema Nähe zu den Menschen in ihrer Pluralität zur Seite gestellt. ${ }^{1}$ Das Thema würde kaum so prominente Deklarationen erfahren, würde es sich um eine Selbstverständlichkeit handeln. Vielmehr zeugen diese Zukunftspapiere zum einen von der Unabdingbarkeit des Themas ${ }^{2}$, zum anderen aber auch von der inzwischen sicherlich weit mehr als unterschwelligen Befürchtung, dass die Institution Kirche die Nähe zu den Menschen verlieren könnte und schon vielerorts verloren

1 Evangelisch-reformierte Kirche im Kanton St. Gallen, St. Galler Kirche 2025. «nahe bei Gott - nahe bei den Menschen», 2019, URL: https://www.ref-sg.ch/aktuelles-details/eine-bunte-vision.html (abgerufen am 11.03.2019); Bistum St. Gallen, Bistum St Gallen auf dem Weg in die Zukunft. Pastorale Perspektiven und Grundhaltungen, 2012, URL: www.bistum-stgallen.ch/fileadmin/kundendaten/Dokumente/HiReWe/3.2.1._Pastorale_Perspektiven.pdf (abgerufen am 11.03.2019).

2 Die evangelisch-reformierte Betitelung liest sich wie eine Paraphrase auf das jesuanische Doppelgebot; gemäss Mk 12,29 immerhin das grösste Gebot. hat. Neben Glaubwürdigkeitsproblemen ist bei der Suche nach Gründen unabweislich auf den Relevanzverlust kirchlicher Verkündigung einzugehen. Mit ihrer Sprache, ihren Formen und ihren Orten gelingt es den Kirchen fortlaufend weniger Menschen mit der heilvollen Wirklichkeit Gottes in Berührung zu bringen.

Gerne wird aber auch immer wieder auf die spezielle Rolle hingewiesen, welche der Diakonie in diesem Spannungsfeld zukommen kann. In der diakonischen Tätigkeit wird in Adaption von Mt 25,40 das entscheidende Kriterium kirchlicher Glaubwürdigkeit in einer säkularen Gesellschaft gesehen ${ }^{3}$, oder in Werken der Caritas können Christen in der Welt göttliches Heil erfahrbar machen. In der Diakonie wird der Kirche Zukunft zugeschrieben, denn in ihr würde sich die Kirche wieder stärker für die Menschen öffnen und so dem kirchlichen Leben Impulse ermöglichen. ${ }^{4}$

Angesichts solcher Einschätzungen ist es beunruhigend, wenn gerade die Diakonie als gefährdetster kirchlicher Dienst bezeichnet wird. So meint Rainer Bücher: «Die Diakonie, also die christliche Nächstenliebe im Ernstfall der Solidarität mit Notleidenden und Unterdrückten, gehört zu den fragilsten der klassischen kirchlichen Handlungsvollzüge.» ${ }^{5}$ Es scheint bisweilen, als ob sich die Kirche die Türe zur eigenen Zukunft selbst zuschlägt.

3 Vgl. Klaus Krämer, Die missionarische Dimension diakonischen Handelns, in: Klaus Krämer / Klaus Vellguth (Hg.) Theologie und Diakonie. Glauben in der Tat (Theologie der einen Welt 3), Freiburg i. Br. 2013, 123-132.

4 Vgl. Peter Neher, Für eine zukunftsfähige Kirche, in: Klaus Krämer / Klaus Vellguth (Hg.) Theologie und Diakonie. Glauben in der Tat (Theologie der einen Welt 3), Freiburg i. Br. 2013, 296-308.

5 Rainer Bucher, «Was Ihr den Geringsten...» Die Kirche und ihre Diakonie, in: ders, An neuen Orten. Studien zu den aktuellen Konstitutionsproblemen der deutschen und österreichischen katholischen Kirche, Würzburg 2014, 179-187, 179.
Jahrbuch Diakonie Schweiz 3 (2019) - ISSN 2504-3994

Dieser Text ist lizenziert unter einer Creative Commons Namensnennung 4.0 International Lizenz (CC BY 4.0): (https://creativecommons.org/licenses/by/4.0/)
Jahrbuch Diakonie Schweiz 3 (2019) http://dx.doi.org/10.22018/JDS.2019.11 
Der CAS Diakonie-Entwicklung ist ein neukonzipierter Lehrgang am Weiterbildungszentrum der Fachhochschule St. Gallen in Kooperation mit der evangelisch-reformierten Kirche im Kanton St. Gallen und dem Bistum St. Gallen. ${ }^{6}$ Nach mehrmaliger erfolgloser Ausschreibung in den vergangenen Jahren wurde er mit der neuen Lehrgangsleitung neu strukturiert und ausgerichtet, so dass er am 1. April 2019 in der neuen Form starten kann. Im CAS Diakonie-Entwicklung initiieren die Teilnehmenden ein konkretes Projekt und üben an ihrer Kompetenz, diakonische Projekte zielführend und effektiv umzusetzen. Sie eigenen sich Fertigkeiten in Teambuilding, Öffentlichkeitsarbeit und Fundraising an, und entwickeln ihr Freiwilligenmanagement weiter. Die Diakonie-Entwicklerinnen werden in ihrer eigenen Institution zu Anwälten der Diakonie und können Kirchgemeinden und Pfarreien unterstützen, im lokalen gesellschaftlichen Umfeld präsenter und verankert zu sein.

Besonders hervorzuheben sind dabei zwei Aspekte, auf welche der CAS Diakonie-Entwicklung hinwirkt. Dies ist zum einen die Vergewisserung, dass die Kirche der Zukunft eine diakonische Kirche sein muss. (1.) Zum anderen ist dies die interdisziplinäre Zusammenarbeit der Fachdisziplinen bei der Aneignung diakonischer Kompetenzen. (2.)

6 Vgl. URL: www.fhsg.ch/diakonie (abgerufen am 11.03.2019). Die Lehrgangsleitung liegt bei den Arbeits- bzw. Fachstellen für Diakonie der beiden Landeskirchen, Maya Hauri Thoma, URL: www.ref-sg.ch/diakonie und Gregor Scherzinger, URL www.caritas-stgallen.ch/Diakonie (abgerufen am 03.04.2019).

\section{Die Kirche der Zukunft muss eine diakonische Kirche sein - warum?}

$\mathrm{Zu}$ Zeiten von kirchlichem Strukturwandel ${ }^{7}$, Mitgliederzahlenrückgang ${ }^{8}$ und Missbrauchsskandal ${ }^{9}$ liegt eine naheliegende Reaktion darin, dass sich kirchliche Institutionen nach innen richten und auf ihr Innenleben konzentrieren. Dies muss sich nicht unbedingt in der Form einer sektenähnlichen «heiligen» Kontrakultur vollziehen, sondern zeigt sich allein auch schon im Bestreben, den eigenen Kern zu konsolidieren, um sich selbst in der nach eigenem Gefühl schliessenden «small world» behaupten zu können. Der Prager Theologe Tomas Halik spricht in diesem Zusammenhang von einer zentripetalen Kirchendynamik. Sie wird von einem Glaubenstyp getragen, der eine institutionelle Heimstatt, also ein gerahmtes und stark abgesichertes Heim für seine Religiosität zu schaffen versucht. ${ }^{10}$ Hält man es aber ebenfalls mit Halik und sieht den Auftrag der Kirche auch darin, «die Welt zu einem besseren Ort für das Leben aller Menschen» zu ma-

Vgl. Schweizerischer Nationalfonds, Die Religiosität der Christen in der Schweiz und die Bedeutung der Kirchen in der heutigen Gesellschaft (NFP 58 Themenheft IV) 2011, URL: http://www.snf.ch/SiteCollectionDocuments/nfp/nfp58/NFP58_Themenheft04_DE.pdf (abgerufen am 10.03.2019).

8 Schweizerische Pastoralsoziologische Institut (SPI), Was passiert mit der katholischen Kirche? Medienmitteilung vom 15. Nov 2018, URL: https://spi-sg.ch/was-passiertmit-der-katholischen-kirche (abgerufen am 10.03.2019).

9 Vgl. bspw. Magnus Striet / Rita Werden (Hg.), Unheilige Theologie. Analysen angesichts sexueller Gewalt gegen Minderjährige durch Priester (Katholizismus im Umbruch 9), Freiburg i. Br. 2019; Simon Hofstetter / Esther Gaillard (Hg.), Heim- und Verdingkinder. Die Rolle der reformierten Kirchen im 19. und 20.Jahrhundert, Zürich 2017; URL: https://www.ref.ch/news/kirchenbund-mag-sich-noch-nicht-bei-missbrauchsopfern-entschuldigen (abgerufen am 08.03.2019).

10 Tomas Halik, Katholizität. Plädoyer für eine Kirche der offenen Rändern: Communio IKaZ 47 (2018), 229-237, 233 
chen, so liegt unweigerlich die Herausforderung bar, die «Anknüpfungspunkte an die säkulare Gesellschaft (des Abendlandes)» zu halten beziehungsweise wohl sie grosso modo erst ausfindig zu machen. ${ }^{11}$ Dazu jedoch braucht es die gegenläufige zentrifugale Bewegung der Kirche. Die Bewegung hin zu den Menschen am Rande und jenseits der sichtbaren Grenzen der Kirche, zu denen, die sich nicht einordnen lassen. ${ }^{12}$ Diesen offenen Charakter der Kirche zu wahren, ist genau eine Eigenheit der Diakonie. Sie ist der Kirchenort, an dem die Institution mit Menschen von draussen in der Welt in Berührung kommen kann. Sie orientiert sich von ihrem Anspruch her genau nicht an einer ideellen Kirchengrenze, sondern will sich durch die Not und das Leiden konkreter Menschen führen lassen. Eine lebendige Diakonie bewahrt folglich vom Kommunikationsabbruch zwischen Kirche und Welt. Sie gibt der Kirche dabei auch die Chance - wenn sie sie nicht gar dazu zwingt - die Welt in ihrer Eigenständigkeit wahrzunehmen, und bewahrt vor religiöser oder theologischer Überdeutung.

Die Bedeutung dieser Kontaktstelle zur Welt ist nicht zu unterschätzen. Nicht zuletzt auch wegen ihrer normativen Orientierung. Will Kirche sich nicht gegenüber der modernen Welt verschliessen beziehungsweise als weltentzogene Kontrakultur fungieren und folglich aber ihre weltmitgestaltenden Potentiale verspielen, dann hat sie sich dem Selbstbewusstsein der Welt zu stellen. Dieses Selbstbewusstsein ist sicher in sich vielschichtig und nur schwerlich auf den Punkt zu bringen, allerdings schein es an einem Ort zusammenzulaufen, nämlich dem gesellschaftlichen Ideal einer möglichst freien, individuell gewährten Lebensführung. Die dominierende Stellung, in welcher sich dieses Ideal ausprägt, zeigt sich auch gerade in Gegenbewegungen, welche sich als Kontrapunkte zum «allseits

11 Tomas Halik, Die Zukunft des Glaubens: Welt vom 31. Mai 2014, URL: https://www.welt.de/kultur/article128570991/Die-Zukunft-des-Glaubens.html (abgerufen am 11.03.2019)

12 Halik, Katholizität (Anm. 10), 231. herrschenden Selbstentwerfungsimperativs» mit seinen Überforderungsphänomenen entwerfen. ${ }^{13}$ Der Komplexität der Multioptionsgesellschaft, welche sich im unausweichlichen Druck bemerkbar macht, den eigenen Lebensentwurf selbst entwerfen zu müssen, wird gerade von religiöser, teils auch kirchlicher Seite her die Einfachheit eines durchgehend religiös normierten Systems als Heilsversprechen entgegengesetzt. Allerdings kann auch diese Unterwerfung unter eine klare Ordnung nicht darüber hinwegtäuschen, dass der Verzicht auf Autonomie in einer sich modern verstehenden Welt sich nichts anderem als einer autonomen Entscheidung verdankt. ${ }^{14}$ Ohnehin wäre eine Grenzziehung zwischen Kirche und Welt ein Kunstprodukt, sind doch längst grosse Teile der sich noch als kirchlich gebunden fühlenden Menschen längst in der Moderne angekommen und agieren selbst nach modernen Grundsätzen. Davon legen gerade viele der innerkirchlichen Konfliktszenarien Zeugnis ab, wo genau Autonomiebewusstsein mit kirchlichem Ordnungsdenken kollidiert.

Was aber haben diese Überlegungen zur Weltausrichtung der Kirche mit den Anliegen der Diakonie zu tun? Wenn dem so ist, dass die Diakonie die Kirche in der Welt verhaftet hält, dadurch dass sie sie immer wieder zur Ausrichtung am in der Welt lebenden Menschen drängt, so ruft sie dadurch auch jeweils den geltenden normativen Bezugspunkt in Erinnerung, welcher gleichzeitig auch wieder das Handeln der Kirche, insbesondere deren Einsatz für eine für alle bessere Gesellschaft orientieren muss:

«Als normativen Bezugspunkt aller Konzeptionen von Gerechtigkeit in der Moderne können wir [...] die Idee der individuellen Selbstbestim-

13 Magnus Striet, Weltliche Welt. Eine fundamentaltheologische Grundlegung, in: Martin Kirschner / Joachim Schmiedl (Hg.), Diakonia. Der Dienst der Kirche in der Welt (Katholische Kirche im Dialog 1), 41-43.

14 Vgl. dazu auch Hans Joas, Glaube als Option. Zukunftsmöglichkeiten des Christentums, Freiburg i. Br. 2012
Jahrbuch Diakonie Schweiz 3 (2019) - ISSN 2504-3994

CC by 4.0
Jahrbuch Diakonie Schweiz 3 (2019) http://dx.doi.org/10.22018/JDS.2019.11 
mung betrachten: Als «gerecht muss gelten, was den Schutz, die Förderung oder die Verwirklichung der Autonomie aller Gesellschaftsmitglieder gewährleistet.» 15

Diese Verzahnung von Gerechtigkeit und individueller Freiheit fordert die Diakonie dazu auf, nicht nur Benachteiligte zu unterstützen und für sie anwaltschaftlich Partei zu ergreifen. Das Helfen muss unter dem Anspruch stehen, die Subjektwerdung Marginalisierter zu ermöglichen. Es verbietet sich, «Menschen zu blossen Objekten helfenden Handelns zu degradieren» ${ }^{16}$. Dies wird in der Diakonie gerne in der Gestaltung von Empowerment-Prozessen umgesetzt. Befähigung und Stärkung der Selbstkompetenz sollen die Teilhabe an und das Teilen von Macht ermöglichen. ${ }^{17}$ Die Asymmetrie in Konstellationen solidarischen Handelns sind kritisch zu reflektieren und es gilt, aktiv zu verhindern, dass helfendes Handeln zu einer verkappten Form von Herrschaft wird. Um diesen Wandel von der Fürsorge hin zu einer autonomieorientierten Assistenz zu verwirklichen, braucht es nicht nur die Selbstreflexion auf ein kooperatives Ethos des Handelns hin, sondern auch Methodenkompetenz, mit der Kooperations- und Partizipationsprozesse erfolgreich gestaltet werden können.

Eine Diakonie, die sich diesem Anspruch stellt, wird nicht darum herumkommen, die beteiligten Menschen nicht nur als Subjekte der Diakonie zu sehen, sondern auch als Subjekte der Kirche. Insofern hat die Diakonie eine Erinnerungsfunktion, mit der sie die Kirche beharrlich mit ihrem Auftrag für Menschen am Rande konfrontiert. Gleichzeitig hat sie diese

15 Axel Honneth, Das Recht der Freiheit - Grundriss einer demokratischen Sittlichkeit, Frankfurt a. M. 2011, 40.

16 Heinz Rüegger / Christoph Sigrist, Diakonie - eine Einführung. Zur theologischen Begründung helfenden Handelns 2011, 210.

17 Vgl. Schweizerischer Evangelischer Kirchenbund, Grundwerte aus evangelischer Sicht (SEK Position 7), Bern 2007, 43-48.
Funktion so einzulösen, dass sich der Kirchenraum erweitert und unter Umständen gar sein Zentrum an die vormals vermeintliche Peripherie verschiebt, denn: «Diakonal fruchtbar wird Kirche nur dann werden können, wenn sie sich selbst diakonisiert, die Welt in sich hinein holt.» ${ }^{18}$ Es könnten so originelle ekklesiogenetische Prozesse erst in den Blick kommen, an vormals unbeleuchteten Orten wie in Sozialprojekten oder karitativen Aktivitäten, welche umgekehrt helfen, Kirche in der heutigen Welt neu zu verstehen. ${ }^{19}$

Mit Blick auf das Weltverhältnis der Kirche liesse sich insofern der Diakonie eine zweifache Aufgabe zuschreiben. Zum einen verweist sie die Konkretisierung des Credos eines menschenwilligen und menschenfreundlichen Gottes in die radikale Erfahrung der Weltlichkeit der Welt und wehrt jegliche spiritualisierte Ver- oder Überdeutung dieser Welt als diesem Credo unangemessen zurück. Zum anderen holt die Diakonie ihrem Anspruch nach die Welt in die Kirche hinein, um Kirche als Kirche in der heutigen Welt neu zu verstehen, aber auch um gleichzeitig die Welt zu einem besseren Ort für die Menschen zu verwandeln.

Dabei ist es nicht leicht, den diakonischen Auftrag der Kirche für die Welt auf einen Punkt zu bringen angesichts dem durch mannigfache Herausforderungen in Frage gestellten Ideal einer gerechteren Gesellschaft. Folgt man allerdings dem normativen Anspruch einer sich reflexiv gewordenen Moderne liesse sich dieser durchaus benennen und so auch als oberstes Korrektiv benützen:

«Die primäre diakonale Aufgabe der Kirche in einer auf Freiheit setzenden, gerade deshalb aber immer fragilen und von Auszehrungen bedrohten Gesellschaft besteht darin, zu einer verbindlichen Übernahme der

18 Striet, Weltliche Welt (Anm. 13), 56.

19 Vgl. Rainer Krockauer, «Wir Leute von der Strasse» - Sozialraumpastoral heute, Beitrag auf feinschwarz.net vom 29. Januar 2019, URL: https://www.feinschwarz.net/wirleute-von-der-strasse-sozialraumpastoral-heute/ (abgerufen am 06.06.19).
Jahrbuch Diakonie Schweiz 3 (2019) - ISSN 2504-3994

CC by 4.0
Jahrbuch Diakonie Schweiz 3 (2019) http://dx.doi.org/10.22018/JDS.2019.11 
Freiheit, zu einer Selbst-Wahl der Freiheit als Freiheit, zu ermutigen.» ${ }^{20}$ Denn ohne diese gelebte Freiheit wird es keine Zivilisation der Gerechtigkeit geben. Einer Kirche, die sich an diesem Bild orientierte, wäre angesichts gegenwärtiger gesellschaftlicher Ambivalenzen durchaus eine gesellschaftlich relevante Rolle zuzutrauen, nämlich selbst «ein Ferment des freieren und sinnvolleren Lebens» zu sein und damit zugleich für die Gesellschaft ein Angebot darzustellen, «dessen diese dringend bedarf und das vielleicht allein von einer Kirche der Freiheit gemacht werden kann.» ${ }^{21}$

Was wäre dieses «Mehn»? Eine Lebenspraxis, die sich bewusst ihr eigenes Leben entwirft und somit die eigene Freiheit ergreift, wird dennoch unweigerlich mit den Grenzen der Realisierbarkeit dieser Freiheit in Berührung geraten. Es mag entlasten und vor der Gefahr der Melancholie bewahren, diesen 〈Zwang〉 zur Selbstgestaltung der Welt als von Got selbst so ermöglicht zu verstehen. Enthält doch der Glaube an den menschenfreundlichen Gott auch das von Gott selbst gegebene Versprechen, dass er «das Geglückte verewigen, das Scheitern versöhnen und da für Gerechtigkeit sorgen wird, wo dies menschlicher Praxis versagt ist.» ${ }^{22}$ Dies mag zum Risiko ermutigen, für mehr Gerechtigkeit einzustehen, und davor bewahren, Gefühle von Vergänglichkeit und Absurdität dominieren zu lassen.

\section{Nötige Fachkompetenzen in einer diakonischen Kirche}

Diese theologischen Weichenstellungen weisen schon aus sich heraus auf die zweite wichtige Überlegung in der Neukonzeption des CAS Diakonie-

20 Striet, Weltliche Welt (Anm. 13), 51

21 So Hermann Krings Worte aus dem Jahr 1972, zitiert nach Magnus Striet, Weltliche Welt (Anm. 13), 47.

22 Striet, Weltliche Welt (Anm. 13), 55
Entwicklung: Die Realisierung einer diakonischen Kirche ist nicht ohne Fachkompetenzen möglich.

Neben der theologischen und diakonischen Selbstvergewisserung legt der CAS Diakonie-Entwicklung deshalb einen zweiten Schwerpunkt auf die Aneignung von Fachkompetenzen in der Gemeinwesenarbeit. Kirche und Theologie haben kein eigenes human- oder sozialwissenschaftliches Sonderwissen über die Zusammenhänge und Gesetzlichkeiten von Gesellschaft und Mensch. Es gilt hier das einfache Gesetz, dass sie ihre Aktivitäten an den aktuellen fachwissenschaftlichen Standards zu messen haben. Wollen Kirche und Diakonie ihr Evangelium eines menschenzugewandten Gottes im Handeln zum Ausdruck bringen und in der Welt Wirklichkeit werden lassen, müssen sie sich in der Gesellschaft und ihren Fachdiskursen bewegen und entsprechende, professionelle Strukturen ausbilden. In der Aneignung der Methoden der Gemeinwesenarbeit steht insbesondere der Perspektivenwandel im Hintergrund, welchen die Kirche in ihrer Seelsorge zu vollziehen versucht: von der Anbieterin zur Begleiterin. Es geht darum, durch die Einübung eines sozialräumlichen Blickes zur Entwicklung in den Raum hineinzuschauen, statt ihn automatisch auf sich zu beziehen.

Die Gründe, weshalb es in den vergangenen Jahren in der Vorgängerkonzeption des CAS immer wieder nicht gelungen ist, eine ausreichende Zahl interessierter Personen zusammenzubringen, welche den Lehrgang dann auch absolvierten, sind sicherlich vielschichtig. Ein gewichtiger Grund jedoch wird auch mit der fragilen Situation der Diakonie im Gesamt der Seelsorge zu tun haben, welche immer wieder zu Effekten der Marginalisierung oder Hintanstellung in der Prioritätenliste führt.

Diakonie muss in der internen Arbeitsaufteilung gerne in Teilzeitstellen und kleinen Zeitpensen abgedeckt werden, gleichzeitig aber wird das diakonische Arbeitsfeld als grenzenlos und prinzipiell nicht zu bewältigen angesehen, was weitere Konnotationen auf den Plan ruft: Die Diakonie kommt immer mit einem schlechten Gewissens daher, weil ihrem Anspruch nach nie genüge getan werden kann. So erscheint das Arbeitsfeld
Jahrbuch Diakonie Schweiz 3 (2019) - ISSN 2504-3994

CC by 4.0 
der Diakonie selten als attraktiv und für die Verantwortlichen befriedigend.

Dieser Gemengelage wurde bei der Neukonzeption des CAS Diakonie-Entwicklung Rechnung getragen. Der Lehrgang soll direkt in die jeweilige diakonische Praxis hineinwirken, und bei der Konzentration der Ressourcen unterstützen. Ebenfalls soll er durch die Integration von Projekttagen vor Ort die knappen Ressourcen schonen beziehungsweise eine Orientierung und Fokussierung der lokalen Diakonie unterstützen. So wird die Rückbindung an den konkreten Betätigungsort und das Arbeitsumfeld der Teilnehmenden als Kursaufgabe integriert, was unter Umständen auch die Teilnahme in einem Teilpensum ermöglicht. Dies wird unterstützt durch das Instrument des direkten Coachings in der lokalen Projektarbeit. Ebenfalls ist in der Projektphase für Teamarbeit, Leadership und Teambildung ein eigener inhaltlicher Block reserviert, um der Gefahr des Alleingängertums in der Diakonie entgegenzuwirken. Hier wird auch Wert auf die Rollenklärung gelegt, damit in der Diakonie Tätige sich zukünftig stärker als Begleitende denn als Subjekte der Diakonie vor Ort verstehen können. Aus dem Grund kommt der Gestaltung von Partizipationsprozessen im Rahmen von Freiwilligenmanagement, Projektplanung und Öffentlichkeitsarbeit eine wichtige Funktion zu. Diakonische Projekte und Aktivitäten sollen den Rollenwechsel vereinfachen. Die Diakonie sol nicht allein als Raum entworfen werden, in dem institutionelle Unterstützung angeboten wird für Menschen in gewissen Bedarfslagen. Die Diakonie soll einen Raum öffnen, in dem Menschen sich selbst aktiv beteiligen. So könnte Diakonie auch an der Einlösung des Anspruchs arbeiten, Kirche nahe bei den Menschen zu halten und Kirche als Raum zu entwerfen, in dem jeder sich nach seiner Art gestalterisch betätigen kann. Die Vision wäre, Kirche als Zukunftsfeld zu sehen, in dem die Gesellschaft an ihrer eigenen, einer besseren Zukunft kreativ arbeiten kann. Partizipationsprozesse würden garantieren, dass einerseits Kirche eine Plattform zur sozialen Gestaltung wird, und dass andererseits Kirche selbst im Alltag und in den Lebenswelten präsent bleiben kann.
Der CAS Diakonie-Entwicklung hofft, motivierte Mitstreiterinnen und Mitstreiter der Diakonie in diese Richtung begleiten zu können.

Autor:

Gregor Scherzinger, Dr. theol., Mitarbeiter der Fachstelle Diakonieanimation bei der Caritas St.Gallen-Appenzell und Lehrbeauftragter fïr Ethik am Religionspädagogischen Institut der Universität Luzern, Lehrgangsleitung des CAS Diakonie-Entwicklung zusammen mit Maya Hauri Thoma, Beauftragte Diakonie der Ev.-ref. Kirche des Kantons St. Gallen, Diakonin, Supervisorin/Coach BSO, Gemeinde- und Organisationsberaterin 